\title{
The impact of energy dissipation to the stability in financial system
}

\author{
Yikui Qiu \\ School of Finance, Central University of Finance and Economics, Beijing, China \\ Email address: \\ qyk_1999@126.com
}

To site this article:

Yikui Qiu. The Impact of Energy Dissipation to the Stability in Financial System. International Journal of Economics, Finance and Management Sciences. Vol. 2, No. 1, 2014, pp. 111-116. doi: 10.11648/j.ijefm.20140201.22

\begin{abstract}
Financial system has a characteristic of dissipative structure theory. In the nonlinear motion process, financial system is extremely easy to produce energy dissipation, which would lead to the emergence of system instability. In this paper, the dissipative structure characteristic of financial system would be analyzed in the first, and the energy function of financial system would be given. Then the energy dissipation and the energy exchange in financial system would be studied. At last, the influence of energy dissipation to system stability would be given. Financial system instability is related to the total entropy and the impact resistance ability of system. We can control system stability by these two factors. The research is importance to the study of financial stability theory and the control of financial system stability.
\end{abstract}

Keywords: Dissipative Structure, Energy Dissipation, System Stability, Financial System

\section{Foreword}

Since 1990s, with the developing of financial globalization and financial liberalization, the financial industry has obtained an unprecedented development. However, it also leaded to numerous financial crises in global scope. Financial crisis restricted the development of economy and society, and bring serious impact to people's life. How did the financial crisis take shape? How to understand and grasp the financial crisis from the root? These questions have already caused an extensive thinking by governments and financial theory researchers. Financial system is an open and complicated system. There is energy dissipation in the motion process. Financial system energy dissipation can lead to system's instability easily, and it can result to financial crisis with the development of system instability.

In the study of financial stability, a lot of scholars have made attempts using dissipative structure theory. Philippatos and Wilson (1972) ${ }^{[1]}$ calculated the optimal portfolio coefficient using the entropy function to instead the variance, and they established the mean-entropy model instead of the mean-variance model, the new model is also applied to the probability distribution. According to the first four moments of the return distribution, Maasoumi and Theil (1979) ${ }^{[2]}$ measured the income by entropy function. Chandra and Singpurwalla (1981) ${ }^{[3]}$ analyzed and discussed the concept of entropy sequence in the economics. In the research of investment portfolio, Andreia (2005) ${ }^{[4]}$ measured the risk using entropy and variance respectively. He compared the differences between them, and got the conclusion that entropy must be more accurate than variance as risk measurement vector. Xuguang Nan (2005) ${ }^{[5]}$ introduced entropy and dissipative structure theory into the study of financial system. He analyzed the entropy effect of financial system, and pointed out that financial fragility is caused by its internal entropy. Lifa $\mathrm{Hu}$ (2009) ${ }^{[6]}$ believed that it would cause the collapse of the virtual economy system and outbreak financial crisis if the activity of system's elements and components is not limited, or the exchange of substance and energy between system and the outside is lacked, or the stability of the system's dissipative structure is destabilized by something in outside. Meng Xu (2011) ${ }^{[7]}$ analyzed financial crisis from the view of dissipative structural theory. She believed that financial crisis is caused by the excessive financial innovation, moral risk, inadequate financial supervision and the real estate market bubble's burst. Xukai Huang (2012) ${ }^{[8]}$ believed that the inverted Pyramid structure of modern virtual financial assets and physical assets caused the entropy increase in financial system. The entropy induced the instability of financial system. When the instability reached certain degree, it would cause financial crisis.

From the research above, we can see that the dissipative structure theory can be widely applied to the study of 
financial system stability. The dissipative structure in financial system is an important factor that leading to the instability of system. But these studies only described the surface phenomenon, they hadn't explained the energy dissipation in financial system, and didn't give energy dissipation function. The specific effects of financial system energy dissipation to system stability hadn't been analyzed clearly. This paper will study these view above. It can enrich financial stability theory, and provide a reference for the control of financial system stability.

\section{The Dissipative Structure Characteristics of Financial System}

In dissipative structure theory ${ }^{[9]}$, there is a view that, by fluctuation mutation, which namely non-equilibrium phase transition, when the external conditions or some parameters of system develop into certain critical value, an open system far from the equilibrium state can develop into a new state structure from the original chaotic state. The new state structure is time order, or space order, or function order. It has some stability, and will not cause upheaval by a tiny perturbation external. As an open complex giant system, financial system has some basic features of dissipative structure. First, financial system is an open system. Financial system is composed by a large number of system components. According to the understanding on environment and environment development prospects, and its intended target, each component makes decision independently. By these, they provide maximum openness for the system. Financial system exchanges material and energy with the financial environment. The exchange is imbalance. The components internal and external are interconnected, interaction, mutual competition, and exist some nonlinear dynamic process. These factors make the financial system is developed into an advanced, unbalanced and orderly structure from the initial disordered structure.

Secondly, financial system is far from equilibrium state. In financial system, the supply and demand of capital has space-time contradiction and non symmetry. From the time level, the subjects of financial market are in the progress of making different capital cycle and turnover. The lie idle and shortage of funds have their own time lag, and can't arise fully synchronous and corresponding. From the space level, the supply and demand of capital also often show uneven distribution at the same time. In the relationship between supply and demand, the power between supply and demand is also unbalanced. They can't remain completely synchronous growth or decline. They even can form an equilibrium price under certain conditions. But the equilibrium is relative rather than absolute, and it is often being broken by various factors. These make the system is always being in an equilibrium and asymmetric state.

Third, financial system has nonlinear interactions. Financial capital has its own characteristics. The supply and demand is often influenced by some factors such as political, economic, cultural, technological, psychological expectations and so on. The way of these factors influence financial system is not simple superposition but mixed nonlinear. These make the system presents a state of height non - equilibrium.

Fourth, financial system has fluctuations trigger and intensification. The fluctuations in financial system can be understood as the fluctuation of some factors that influencing the system's development. In general, elements remain relatively constant or change a little in macro. But in microscopic point, the fluctuation is frequently emergence because of the influence of various complicated factors inside and outside. So there is some random fluctuation in financial system. When financial system is being in equilibrium state, the fluctuation triggered by the micro fluctuation in financial system is not enough to break the original order for the system's stability. But when the system is in a critical point, the micro fluctuation will be quickly put into large by nonlinear interactions and chain effect. It will produce a macro fluctuation and lead to the catastrophe of system. It will build a new system order from the unstable state. Then it will form a new dissipative structure.

\section{The Energy Dissipation and Energy Exchange in Financial System}

Financial system is an open system. In the process of system operation, there is a constant exchange of matter, energy and information between every part of the system and the external financial environment. These form a material flow, an energy flow and an information flow in time and space. The material flow is consisted of the human resources flow, the site and equipment's transfer, the financial instruments and so on. It is driven by the competition and cooperation among financial system elements, and the exchange between financial system and financial environment. The energy flow refers to the amount of value flow in the system. It can be expressed by the form of monetary price volatility, the flow of funds and so on. It is often flowed with the help of monetary form. In financial system, there is mass of complex information. Through the process of collection, transmission, processing, storage, retrieval, analysis, all kinds of information are transported and exchanged continuous, and be merged into the information flow. The conduction of information flow depends on the flow of material and the flow of energy. The material flow, the energy flow and the information flow often interweave together, form an energy of financial system, and flow in the form of monetary funds, and achieve the exchange by the forms of funds credit flow and monetary circulation between the internal elements and external financial environment, so as to realize the transformation of financial system energy and the conversion of system state.

\subsection{The Energy Dissipation of Financial System}

According to the second thermodynamics law, various 
functional transformations occurred in the nature constantly is irreversible. In the irreversible process, the energy is degenerating continuously. It becomes into useless energy which can't be used to do work. In the conversion process, financial system can produce energy dissipation too. It will be converted into useless energy. We can analyze these question by the energy expression in chaotic system presented by Sarasola and others ${ }^{[10,11]}$.

First, we suppose the equation of financial system is:

$$
\dot{x}=f(x)
$$

Among them, $x=\left(x_{1}, x_{2}, \cdots, x_{n}\right), \dot{x}=\left(\dot{x}_{1}, \dot{x}_{2}, \ldots, \dot{x}_{n}\right) \in R^{2}$, $f: U \rightarrow R^{n}$ is a smooth function, $U \subset R^{n}$. The kinetic equation can be expressed as a generalized Hamiltonian form:

$$
\dot{x}=M(x) \nabla H
$$

Among them, $H(x)$ is Hamiltonian function. It says the smooth energy function of financial system; $\nabla H$ is the gradient vector of $H(x)$. For Hamiltonian systems, $M(x)$ is a skew symmetric matrix, i.e.:

$$
M=\left[\begin{array}{rr}
0 & I_{d} \\
-I_{d} & 0
\end{array}\right]
$$

Among them, $l_{\mathbb{a}}$ represents the unit matrix in $R^{n}$.

For the generalized Hamiltonian systems, $M(x)$ is no longer a skew symmetric matrix, which can be expressed as a plus of a skew symmetric matrix $A(x)$ and a symmetric matrix $R(x)$. Thus, type (2) can be transformed into:

$$
\dot{x}=[J(x)+R(x)] \nabla H
$$

As for the skew symmetric matrix $J(x)$, we know:

$$
\nabla H^{T} J(x) \nabla H=0
$$

Therefore, the derivative of financial system energy delay along the dynamics trajectory is:

$$
\dot{H}=\nabla H^{T} \dot{x}=\nabla H^{T} f=\nabla H^{T}[J(x)+R(x)] \nabla H=\nabla H^{T} R(x) \nabla H
$$

The energy changes of in financial system can be reflected from the volume changes of dynamics system phase space. Therefore, the limiting condition can be introduced into the energy function. According to Liouville theorem, the phase space volume change rate of the velocity vector field $f$ is related to the divergence of the vector field. I.e.:

$$
\frac{d V(t)}{d t}=\int_{A(t)} \operatorname{divf}(x) d x=\int_{A(t)}\left(\sum_{i=1}^{n} \frac{\partial f_{i}}{\partial x_{i}}\right) d x
$$

Among them, $A$ is a closed set in phase space $R^{n}, V$ is the volume of it. Due to the introduction of the foregoing restrictions, namely financial system energy change delaying the trajectories in any time variations is caused by the dispersion component, if we can decompose the scatter components from the vector field $f$ explicitly, the corresponding energy of the vector field $f$ can be determined.

Helmholtz theorem ${ }^{[12]}$ ensures that the vector field $f$ can be decomposed into a vector $f_{e}$ which includes all the rotation part and is irrelevant with the divergence, and a gradient vector field $f_{\mathbb{a}}$ which includes all the divergence part. I.e.:

$$
f(x)=f_{c}(x)+f_{d}(x)
$$

This shows that, the energy change of the system move along the trajectory is only contributed by $f_{\boldsymbol{z}}$. By this we can derive the energy function. Putting the conditions

$$
R(x) \nabla H=f_{d}(x)
$$

into type (6), we can obtain:

$$
\dot{H}=\nabla H^{T} f_{d}(x)
$$

This is the energy dissipation that caused by the dispersion component in velocity vector field.

According to type (9), type (4) can be converted to:

$$
\dot{x}=[J(x)+R(x)] \nabla H=J(x) \nabla H+f_{d}(x)
$$

Combined type (1) and (8), we can get:

$$
J(x) \nabla H=f_{c}(x)
$$

Because $J(x)$ is a skew symmetric matrix, so we can get:

$$
\nabla H^{T} J(x) \nabla H=0
$$

or

$$
\nabla H^{T} f_{c}(x)=0
$$

By the partial differential equation, we can obtain the energy function $H(x)$ of financial system consisted of phase space variables.

\subsection{The Energy Exchange of Financial System}

If the financial system in some country is an isolated autonomous system, i.e. it only exchanges energy with external financial environment, while not produce synchronous resonance with other countries' financial systems. At this point, the derivative (10) of financial system energy metric the energy exchange between system and outside financial environment. It can be understood as an energy dissipation process occurred in the divergence components. The energy dissipation can be positive or negative, which means that the energy exchange between the financial system and financial environment is an input process in sometimes and is an output process in other times. If financial system is an autonomous chaotic system which is set outside of the intrinsic attractor initially, it will experience a process of absorption or dissipation energy. By 
this, it will get into its intrinsic attractor region. After that, there still present reciprocating energy exchange with the outside, but the average net energy changes will be zero. This is because the attractor will return to a state which is arbitrarily close to a state in certain time before. So it also can achieve arbitrarily energy value close to before. Therefore, the average time of the isolated autonomous financial system's energy change is zero:

$$
\left\langle\left[\nabla H^{f}(x)\right]^{T} f_{d}(x)\right\rangle=0
$$

Among them, $<>$ represents the average time, $\boldsymbol{H}^{f}$ represents the energy function of financial system.

However, in reality, financial system is not only exchange energy with external financial environment, but also it can produce synchronous resonance with other countries' financial systems. So, the average net energy changes will not be zero. If the financial system of two countries is a drive-response coupled chaotic system liking the following:

$$
\left\{\begin{array}{l}
\dot{y}=g(y) \\
\dot{x}=f(x)+K(y-x)
\end{array}\right.
$$

Wherein, $\dot{y}=g(y)$ says drive system, $\dot{x}=f(x)$ says the driven system. $K$ is a diagonal matrix, it represents the coupling mechanism between two systems, the diagonal elements are represented by $k$ and $k>0$, it represents the coupling gain. Because the driven system $\dot{x}=f(x)+K(y-x)$ is still a chaotic attractor, it will return to an arbitrarily state close to certain time before, so the average net energy's change in the whole is zero. i.e.:

$$
\left\langle\left[\nabla H^{f}(x)\right]^{T}\left[f_{d}(x)+K(y-x)\right]\right\rangle=0
$$

So we can get:

$$
\left\langle\left[\nabla H^{f}(x)\right]^{T} K(y-x)\right\rangle=-\left\langle\left[\nabla H^{f}(x)\right]^{T} f_{d}(x)\right\rangle
$$

This means that the driven chaotic attractor has been forced to move to the outside of its intrinsic attractor region. The average net energy exchange between financial system and the external financial environment calculated by formula (10) will not be zero. But the energy dissipation of this part will be compensated by coupling mechanism. The energy of the coupling mechanism need to be provided is:

$$
P(k)=-\left\langle\left[\nabla H^{f}(x)\right]^{T} f_{d}(x)\right\rangle
$$

It can be divided into two kinds of situations:

(a) The two countries' financial systems are chaotic oscillator synchronization with identical parameters. At this time, the coupling oscillators have the same dynamic characteristic. Their intrinsic attractor regions are consistent. Therefore, to make their financial system close in phase space, it only needs to provide energy in began. In the process, the change of net average energy is not zero. They need a coupling mechanism to get the energy compensation.
When the two countries' financial systems achieve complete synchronization, both the intrinsic kinetic structure and the locations in phase space are consistent. It is similar to a solitary autonomous system. So they no longer need a coupling mechanism to provide energy basically. Therefore, the synchronization process can be divided into two stages: in the first stage, the coupling mechanism provides energy to force the oscillator moving, so their distance is coincident in the phase space; in the second stage, they are almost not need the coupling mechanism to provide energy. They only need to overcome the inevitable error for the reason of precision in the first stage.

(b) The two countries' financial system is chaotic oscillator synchronization with different parameters and the intrinsic parameters are heterogeneity. At this time, the two countries financial systems achieve the ideal synchronization only when the coupling strength tends to infinity. In practice, the complete synchronization can't be achieved. So there are some problems to discuss the synchronization process in some degree. This synchronization process can be divided into two stages: in the first stage, for forcing the oscillator moving, they need the coupling mechanism to provide large energy, so their location is close in the phase space; in the second stage, due to the intrinsic dynamics of their financial systems are different, so we need the coupling mechanism to provide energy continuously, to compensate the additional energy dissipation bring by kinetic differences. In particular, for the system has different tensile and folding characteristics when being located different positions or different directions in phase space, so the energy compensation need is different.

\section{The Influence of Financial System Energy Dissipation on System Stability}

Financial system has dissipative structure. Due to the existence of the capital virtual and the "Butterfly Effect", the system's stability is very easy to be broken. Financial system needs to exchanges energy with the outside world constantly. By this, it can maintain the relatively stable structure. When the rhythm is disturbed, for example, excessive financial innovation make the financial system away from equilibrium, some characteristics of dissipative structure will not existent, financial system will collapse and cause financial crisis.

The influence of energy conversion the stability in financial system can be explained by the entropy theory. Prigogine (1969) pointed out that ${ }^{[9]}$, for an open system which has substance and energy exchanges with the outside world, the entropy change $(d S)$ can be divided into two parts: one part is the entropy in system due to the irreversible process, it is the entropy production $\left(d_{i} S\right)$, this is always positive; the other part is the entropy $\left(d_{e} S\right)$ caused of the exchanging material and energy by the system boundary, this can be positive or negative or zero. So the total entropy of the system is: 


$$
d S=d_{i} S+d_{e} S
$$

The dissipative structure theory indicates, to improve the system's order, we must control the system's entropy. The necessary conditions is that the system discharge entropy or absorb negative entropy from the outside, that is $d_{\varepsilon} s<0$, and meet $\left|d_{\varepsilon} s\right|>d_{i} s$. Open system can exchange energy and material with the outside world. So when dissipating energy (produce entropy) continuous, it also get the energy from outside (absorb negative entropy). In the process of movement, the system gets develop space constantly.

In financial system, the financial activity is irreversible, and the financial power move change constantly. Because the flow of resources is chaos, the flow level is disorder and there is financial environment friction, in the process of financial resources transfer energy, it will produce energy consumption and form financial entropy. The formula of financial system entropy can be expressed as follows:

$$
d S_{f}=\left[d_{i} S_{f}^{(1)}+d_{e} S_{f}^{(1)}\right]+\left[d_{i} S_{f}^{(2)}+d_{e} S_{f}^{(2)}\right]
$$

Among them, $d S_{f}$ is the total entropy in financial system, $d_{i} S_{f}^{(2)}$ and $d_{s} S_{f}^{(1)}$ represent the internal entropy caused by nonlinear mechanisms in financial system and its external negative entropy respectively, $d_{i} S_{f}^{S 2]}$ and $d_{g} S_{f}^{[2]}$ say the internal entropy and the external negative entropy respectively caused by the resonance or conduction of other financial systems. The internal entropy is caused by financial management risk, asymmetric information, technical level's lack, high competition pressure, the lack of the credit system, the capital market problems, the exchange rate and interest rate policy is not conducive and some factors that is stiffness to the development of financial system. The internal entropy hinders the development of financial system, makes the system more disorderly. The external negative entropy is got from the progress of science and technology, moderate and effective supervision, sound macroeconomic environment and mature capital market. The introduction of external negative entropy can ease the contradictions within the financial system.

Unified the internal entropy and the external negative entropy, we can get:

$$
d S_{f}=\left[d_{i} S_{f}^{(1)}+d_{i} S_{f}^{(2)}\right]+\left[d_{e} S_{f}^{(1)}+d_{e} S_{f}^{(2)}\right]=d_{i} S_{f}+d_{e} S_{f}
$$

Among them, $d_{t} S_{f}$ is the internal entropy accumulated, $d_{z} S_{f}$ is the external negative entropy flow accumulation. This is the total entropy formula of financial system.

The internal entropy expands the risk of financial system, and makes a serious impact on the stability of financial system. But through technological innovation, the development of market, the implementation of appropriate and effective regulatory, financial system can certain negative entropy obtain from the external financial environment. It will reduce the financial risk to a certain extent, and improve the ability to resist risks. In the process of the total entropy changing, the disorder degree and the confusion degree of financial system change unceasingly, the instability of system follow the same changing constantly. When $\left|d_{i} S_{f}\right| \geq\left|d_{z} S_{f}\right|$, the internal entropy is larger than the external negative entropy flow, the total entropy system $d S_{f} \geq 0$. It means that the confusion degree of financial system increases, the stability of financial system decreases. When $\left|d_{i} s_{f}\right| \leq\left|d_{\varepsilon} s_{f}\right|$, the internal entropy is smaller than the external negative entropy, the total entropy of system $d S_{f} \leq 0$, this means that the order of financial system increases, the system stability enhancement. Therefore, from the view of dynamic point, the source of instability in financial system is the internal entropy. The expansion of instability is a macroscopic manifestation of total entropy increase. There is a certain function relationship between the total entropy $\left(d S_{f}\right)$ and the system frangibility $(F)$, namely $F=F\left(d S_{f}\right)$. This function is an increasing function, so the uncertainty of financial system increases with the increase of the total entropy, and decreases with the decrease of the total entropy.

Financial system instability $(F)$ is related to the total entropy $\left(d S_{f}\right)$ and the impact resistance ability of system $\left(\boldsymbol{A}_{\tilde{R}}\right)$. In general, the scale of financial system is larger, the structure is more stable, the system design more perfect, the environment is more security, the anti impact capability of financial system is greater. Therefore, the fragility of financial system $(F)$ can be defined as a rate of the total entropy $d S_{f}$ and the anti impact capability $A_{R}$ :

$$
F=\frac{d S_{f}}{A_{R}}
$$

From the equation above, the fragility of financial system is not only related to the system itself frangibility entropy $F$, but also related to the total entropy of system $d S_{f}$ (including the internal entropy $d_{i} S_{f}$ and the external negative entropy $d_{g} S_{f}$ ), and the anti impact ability of the system $A_{R}$ (including the scale of system $G$, the evaluation index of system structure $J$, the evaluation index of system $Z$, the environmental evaluation index $H I$ and so on). If $T$ is used to express the fragility of financial system, the describing function can be expressed as:

$$
T=T\left[F ; d S_{f}\left(d_{i} S_{f}, d_{e} S_{f}\right) ; \mathrm{A}_{R}(G, J, Z, H I)\right]
$$

\section{Conclusion}

Financial system is a complex giant system. With the development of financial liberalization and financial globalization, the system has become more and more complicated. On one hand, the changing of financial system complex increased the anti impact ability of the system. The changing of financial system complex increased the financial innovation, improved financial efficiency, set up a more effective financial regulation. So it reduced the frangibility of financial system in certain extent. On other hand, the changing of financial system complex also 
expanded the system frangibility entropy. The increase of the system structure complexity, the system functional complexity and the system dynamic complexity made financial system need to consume more energy. This leaded the energy dissipation become larger and larger. So the entropy in financial system is larger and larger. The environment complexity not only increased the internal entropy, but also reduced the negative entropy in the environment to a certain extent. In the rapid complex developing process, financial system accumulated a huge frangibility. It made a security threaten to the system. So the financial crisis occurs often. By constructing the function of energy dissipation, this paper analyzes the energy exchange and the energy dissipation in financial system, analyzes how leading to the frangibility entropy in the system, and analyzes the impact on financial system stability. The research enriches the system stability theory, builds a new way to analyze the system stability.

\section{References}

[1] George C. Philippatos, Gharles J. Wilson. (1972). Entropy, marker risk, and the selection of efficient portfolios, Applied Economics, Issu 4, pp. 209-220.

[2] Maasoumi E., Theil H.(1979). The elect of the shape of the income distribution on two inequality measures, Economics Letters, Issu 4, pp. 289-291.

[3] Chandra M. T., Singpurwalla N. D.(1981). Relationships between some notions which are common to reliability theory and economics, Mathematics of Operations Research, Issue 6, pp. 113-121.
[4] Andreia Dionisio, Rui Menezes, Diana A M.(2005). Uncertainty analysis in financial markets: can entropy be a solution? pp. 8 .

[5] Xuguang Nan.(2005). The dynamic mechanism of financial fragility: financial entropy and institutional change, Journal of Chongqing Radio \& TV University, China, Vol. 17 , Issue 2, pp. 42-44.

[6] Lifa Hu.(2009). The dissipative structure of virtual economy system and the financial crisis in America, Modern economic research, China, Issue. 3, pp. 40-44.

[7] Meng Xu. (2011). The study on financial crisis analyzed by dissipative structure theory, The economy of division, Issue. 3, pp. $42-44$.

[8] Xukai Huang. (2012). Financial fragility, financial innovation, financial risk and financial crisis -- Analysis financial instability based on the entropy theory, China securities and futures, Issue. 6, pp. 207-208.

[9] I. Prigogine. (1980). The complexity evolution and the natural law, The problem of the philosophy of natural science, pp. 3 .

[10] C. Sarasola, F. J. Torrealdea, A. d'Anjou, A. Moujahid, M. Graňa. (2004). Energy balance in feedback synchronization of chaotic systems. Phys. Rev. E, Issue. 69: 011606.

[11] C. Sarasola, A. d'Anjou, F. J. Torrealdea, A. Moujahid.(2005). Energy-like functions for some dissipative chaotic systems. Int. J. Bifurcation Chaos Appl. Sci. Eng., Issue. 15, pp. 2507.

[12] W. K. H. Panofsky, M. Phillips. (1962). Classical electricity and magnetism (Addison-Wesley series in physics). MA: Addison-Wesley, Reading. 